\title{
Clima institucional administrativo de la organización educativa escuela de educación de Colombia
}

\author{
Administrative institutional climate of the educational organization escuela \\ de educación de Colombia
}

\author{
Rosa Rodríguez Guillén \\ rosarodriguezesesco@hotmail.com \\ Escuela de Educación en Colombia, ESESCO, Colombia
}

\section{RESUMEN}

La presente investigación tiene el propósito de describir el clima institucional administrativo de la organización educativa Escuela de Educación de Colombia (Esesco). La investigación de tipo descriptiva, con un diseño de campo transaccional contemporáneo. La población la conforman 19 empleados de la institución estudiada. Para la recolección de los datos se empleó la técnica de la encuesta mediante el instrumento escala Clima Institucional Administrativo (CIA), contentiva de 24 ítems de alternativas de respuestas dicotómicas; la validez se calculó mediante el juicio de experto y una confiabilidad de 0,97 de Alfa de Cronbach. En el análisis de los resultados se utilizó la estadística descriptiva, específicamente la frecuencia absoluta y porcentual, y la mediana como medida de tendencia central por cuanto la variable se midió en un nivel ordinal. Los resultados indican que en el evento existe un clima institucional administrativo desfavorable, ubicándose según el baremo en el criterio desfavorable y lo cual se manifiesta como una debilidad para dicha organización. Se recomienda a la organización educativa Escuela de Educación de Colombia crear espacios en un clima armonioso que propicie la integración del personal, así como afianzar las relaciones interpersonales.

Palabras clave: Cambio, Clima institucional administrativo; conflicto, dirección; formación

\section{ABSTRACT}

The present investigation has the purpose of describing the administrative institutional climate of the educational organization Escuela de Education de Colombia (Esesco). The investigation is of descriptive type, with a contemporary transactional field design. The population consists of 19 employees of the studied institution. The survey technique was used to collect the data using the Institutional Administrative Climate (IAC) scale instrument, containing 24 items of alternative dichotomous responses; the validity was calculated by expert judgment and a reliability of 0.97 calculated with the Cronbach's Alpha. In the analysis of the results, descriptive statistics, specifically the absolute and percentage frequency, and the median as a measure of central tendency were used. The results indicate that the institution has an unfavorable administrative institutional climate, which manifests itself as a weakness for said organization. It is recommended that the educational organization Escuela de Education de Colombia create spaces in a harmonious climate that fosters the integration of personnel, as well as strengthen interpersonal relationships.

Key words: Administrative institutional climate; conflict; direction; change; training 
INTRODUCIÓN

La presente investigación abordó el clima institucional administrativo de la organización educativa Escuela de Educación de Colombia, con la finalidad de describir cómo era el ambiente en la institución estudiada. Entendiendo que clima institucional administrativo es el ambiente generado en una institución educativa a partir de la convivencia cotidiana con el personal administra-tivo que cumple funciones gerenciales de la empresa.

Este ambiente incluye factores sobre el proceso de dirección en la institución, el desarrollo del talento humano, las condiciones de trabajo adecuado, el flujo de la información, la apertura al cambio y la solución de conflicto. Dichos factores son elementos primordiales que ayudan a mantener un buen clima institucional administrativo y son favorables para el funcionamiento eficiente de las organizaciones.

Estudiar el clima institucional administrativo en la organización educativa Escuela de Educación de Colombia, constituye una acción significativa indispensable para el logro de un clima de trabajo adecuado, que pueda favorecer a la corporación en estudio y a todas las instituciones educativas que desean mejorar su clima institucional para el logro de la calidad educativa.

Los desafíos que impone la sociedad del conocimiento en el tránsito de la cultura desde la modernidad hacia la postmodernidad, obligan a las organizaciones a repensar la gerencia como una guía para valorar la condición humana en el tejido relacional del sistema gerencia, individuo, organización y sociedad.

De esta forma, la gerencia de las organizaciones según Lanz (2004, p. 18) "transita un cambio cultural hacia una nueva visión de los contextos organizacionales que validan un paradigma en construcción para pensar en presente".

Siendo así, los paradigmas gerenciales postmodernos no son más que modelos de gestión epocales para pensar y conducir la vida de las organizaciones sociales, como bien lo refiere Salas (2002), cuando expone que los paradigmas gerenciales son el conjunto de conocimientos, procedimientos y acciones que se aplican para ejecutar una política institucional y social mediante la dirección, coordinación, planificación y control, de las actividades de la organización.

Consecuentemente, los procesos gerenciales son implícitos para cualquier organización, sin importar sus fines o características particulares, de allí la importancia de pensar que la gerencia en las organizaciones educativas, debe permitir integrar convicciones, estilos, motivaciones, liderazgos y comportamientos. Al respecto, Balza (2010), expresa que la gerencia educativa debe perfilar la visión del gerente respecto a la valoración de la condición humana y el concepto de compromiso frente a la sociedad y a la misma organización.

Por su parte, la Unesco (1995), expone que la gestión de la educación centra su importancia en los procesos de transformación institucional y de ajuste de la organización escolar a la reforma del estado y a la democratización de las sociedades. Es decir, se procura avanzar más allá del señalamiento de los atributos de diseño más adecuados, para identificar y subrayar los aspectos problemáticos que se vinculan con su implementación.

Según Soto (2008), la gerencia educativa puede concebirse como el proceso, a través del cual se orienta y conduce la labor docente y administrativa de la escuela, y su relación con el entorno, con miras a conseguir los objetivos institucionales, mediante el trabajo de todos los miembros de la comunidad, a fin 
de ofrecer un servicio de calidad, coordinar las distintas tareas y funciones de los miembros hacia la consecución de proyectos comunes.

Hamel y Breen (2008), exponen que en las instituciones educativas la gerencia debe dar respuesta a las tareas de acuerdo a su importancia: establecer objetivos, incentivar y motivar a las personas para lograr su cumplimiento, controlar y coordinar diversas actividades, además de asignar recursos, equilibrar y coordinar los intereses de los actores interesados, construir relaciones sociales, así como aplicar el conocimiento en las diversas áreas que lo requieran.

Igualmente, en las instituciones educativas existen diferentes factores que pueden afectar su desarrollo como es el referido a las percepciones de la comunidad educativa frente al ambiente de trabajo, lo cual incluye las relaciones interpersonales de sus miembros. Uno de estos factores corresponde a la definición de un ambiente o clima de trabajo, el cual afecta los procesos de comunicación interpersonal, la motivación y el cumplimiento de los objetivos institucionales.

De esta forma, las inconsistencias en las relaciones entre los docentes, administrativos y directivos de una institución educativa dan lugar a un clima institucional desagradable, lo cual se refleja en una falta de compromiso del docente frente a su labor de enseñanza aprendizaje y por ende afecta el proceso administrativo y pedagógico de la organización.

Al respecto, Taboada (2006), quien investigó acerca del clima organizacional y el desempeño docente de instituciones educativas pertenecientes a Fe y Alegría en el cono sur de Lima, al explorar acerca de los valores y la ética comportamental del clima organizacional y el desempeño docente en el proceso de enseñanza aprendizaje concluyó que el sistema imperante del clima organizacional existente en las instituciones educativas hace que los docentes asimilen los valores y asuman una conducta ética en su desempeño en las aulas, lo cual hace que el clima de la institución se convierta en una guía para las acciones docentes, con respecto al fortalecimiento de la identidad, estructura y demás características institucionales.

Consecuentemente, el clima institucional en una institución educativa se refiere a la disposición del personal, en todas las áreas y a todos los niveles de la organización, de asumir de manera decidida y permanente la promoción de mejoras en las actividades de liderazgo, disposición para la solución de problemas, creatividad, disciplina, solidaridad y pertinencia que desempeña como miembro de la organización. Se observa de esta manera que el clima institucional se refiere a un ambiente general de la organización.

Sin embargo, en esta investigación se estudió el clima institucional referido a lo administrativo, por cuanto incluía la administración de los recursos materiales, financieros y humanos de los cuales disponía la institución; se incorporó en este aspecto las relaciones laborales, control escolar y cumplimiento de la normativa, es decir, el ambiente que se da con respecto a las actividades administrativas y de interacción social en una organización educativa.

Sobre este particular, Kahr (2010), expresa que el clima institucional administrativo se genera a partir de las vivencias cotidianas de sus miembros. Este ambiente tiene que ver con las actitudes, creencias, valores y motivaciones que tiene cada trabajador y se expresa en las relaciones personales y profesionales.

De esta manera, la satisfacción del personal en el entorno laboral y su percepción del clima institucional administrativo se proyecta en el quehacer diario de la institución. Al respecto, Xiaofu y Qiwen 
(2007), expresa que la percepción del clima incide en la calidad de la educación, la estabilidad emocional en la relación docente estudiantes, así como su vivencia moral y salud mental. En términos de estos autores, la labor docente representa un alto grado de influencia dentro del clima institucional administrativo.

En este sentido, Brunet (2005), expresa que las instituciones educativas que fomenten un clima institucional administrativo favorable con una orientación a la planificación, trabajo en equipo, incorporación de innovaciones, atención de los cambios internos y externos, conseguirán una favorable comunicación, participación, confianza y respeto de los miembros de la organización.

En Colombia, la valoración del clima institucional administrativo en las corporaciones educativas, es un elemento esencial para desarrollo de su estrategia organizacional porque posibilita a los gerentes y recurso humano administrativo la comprensión de lo que se vive al interior de la institución.

Para Davis y Newtrom (1999), el clima institucional administrativo determina la forma en que un individuo percibe su trabajo, su rendimiento, su productividad, su satisfacción. Sin embargo, el tema no es muy estudiado en este contexto, por lo que la Escuela de Educación de Colombia (Esesco), como organización educativa que cuenta con un proyecto educativo acorde con las políticas nacionales, con una imagen corporativa posicionada en el entorno por darle respuesta a los intereses de las comunidades a las cuales se debe, tuvo la inquietud de conocer si el clima institucional administrativo que ofrecía permitía que sus empleados estuviesen articulados con los propósitos e ideales organizacionales.

En este sentido, la Escuela de Educación de Colombia (Esesco), percibió debilidades en los resultados y metas de su trabajo referido a conductas independentistas, poco acordes a los objetivos y metas de la corporación, lo cual, se reflejaban en la conducta poco armoniosa de sus integrantes, y en una comunicación no tan clara en el momento de informar sobre los lineamientos para desarrollar los proyectos. Además, la toma de decisión se realizaba de manera aislada y confusa en la mayoría de los puestos de trabajo.

Igualmente, en los últimos años la organización ha estado afectada por una carencia de liderazgo efectivo, los empleados muestran un marcado comportamiento de falta de motivación y desarrollan actitudes que no llevan al logro de los objetivos institucionales, personales y profesionales. Sin duda, esta situación afecta la política educativa de una canasta educativa integral con estándares de calidad, eficiencia y equidad.

De igual forma, la gerencia $y$ administración de la institución carecía de líneamientos gerenciales claros que los condujera al éxito institucional. La formación gerencial de sus directivos era muy escasa, lo cual ocasionaba que los empleados disminuyeran su productividad y en ciertas áreas de trabajo se presentaban conflictos entre los grupos, asimismo, la empresa no poseía planes prospectivos que configuraran su futuro

En la organización educativa Escuela de Educación de Colombia (Esesco), se cumplía con los requerimientos y normas, pero la efectividad de éstos no se hacía realidad una vez que eran aplicados, lo cual conllevaba a discrepancias entre el deber ser y la realidad, por lo que la posibilidad de alcanzar el logro de la calidad y eficiencia administrativa que garantizan una mayor productividad no se alcanzaba.

De acuerdo con los planteamientos señalados en los párrafos anteriores se 
enunció la siguiente pregunta de investigación con el objetivo de conocer: ¿Cómo era el clima institucional administrativo de la organización Educativa Escuela de Educación de Colombia (Esesco)?

El ámbito mundial obliga a fijar la mirada en la gerencia para buscar lograr un mejor clima en las organizaciones a fin de captar la consecución de sus fines. Según Goncalves (2001), el clima es un fenómeno interviniente que media entre los factores del sistema organizacional $y$ las tendencias motivacionales que se traducen en un comportamiento que tienen consecuencias sobre la organización. Para Gento (1994), el clima o ambiente de trabajo constituye uno de los factores determinantes y facilita no sólo el proceso organizativo de gestión, sino también de organización y cambio.

Ante esto, la gerencia moderna centra su atención en el estudio del ambiente laboral desde el concepto de clima organizacional. Así, Litwin y Taguiri (1969) consideran el clima organizacional como la calidad perdurable del ambiente interno de una organiza-ción que es experimentado por sus miembros, influye en su comportamiento y puede ser descrita como valores de un conjunto particular de características o actitudes de una organización.

De forma similar, Taguiri (1969), vuelve a analizar el constructo clima organización considerándolo como la calidad de una organización que es experimentada por sus miembros y la forma como ésta influye en su comportamiento.

Igualmente, Campbell y otros (1970), consideran el clima organizacional como un conjunto de atributos específicos de cada organización y puede ser incluido por la forma en que la propia organización trata a los miembros de su entorno.

Por último, en lo que se refiere a esta perspectiva Litwin y Stringer (1968) consideran el clima organizacional como un filtro por el cual pasan los fenómenos objetos (estructuras, liderazgo, toma de decisiones), por lo tanto, evaluando el clima organizacional se mide la forma como es percibida la organización.

Las características del sistema organizacional generan un determinado clima organizacional. Esto repercute sobre las motivaciones de los miembros de la organización y sobre su correspondiente comportamiento. Este comportamiento tiene obviamente una gran variedad de consecuencias para la organización como, por ejemplo, productividad, satisfacción, rotación y adaptación, entre otros.

En las últimas décadas del siglo XX se han ejecutado numerosos estudios referidos a la influencia que el concepto del clima organizacional ha alcanzado en las organizaciones.

De esta manera, Molocho (2010), realizó un trabajo donde estudió la influencia del clima organizacional en la gestión institucional. Los resultados arrojados expresaron que el potencial humano influye sobre la gestión institucional de la sede administrativa de la UGEL № 01 Lima sur, con lo que se cumplió con la hipótesis planteada en la investigación. Asimismo, la confianza en el clima institucional correspondiente al diseño organizacional influyó sobre la gestión institucional de la sede administrativa de la UGEL № 01 Lima sur, con lo que también se cumplió con la hipótesis planteada.

Igualmente, Alvares (2017), en una investigación sobre el clima institucional como factor en el rendimiento escolar, obtuvo como resultado que la gestión era determinante en los procesos que se llevaban a cabo en las instituciones educativas, por cuanto, las decisiones de carácter administrativo eran claves para que la parte operativa fuera aplicada de manera correcta, 
con el fin de evitar que pudiera existir inestabilidad en el liderazgo. Además, se percibió que la gestión tenía como cabeza visible el deber funcional que realizaban los directivos, así como la importancia de la participación de toda la comunidad educativa.

Ambas investigaciones revelaron la importancia del clima organizacional, además de explicar que el gerente educativo era la cabeza de las organizaciones. Los resultados arrojados en las encuestas indicaron que se debía revisar detenidamente los elementos que eran transcendentales en la eficacia de los procesos que eran llevados a cabo en las instituciones educativas.

Sin embargo, a pesar de la importancia que se le ha atribuido al estudio del clima organizacional, no existe un aspecto generalizado sobre su significado y alcance. Es más, desde que este tópico despertó interés entre los estudiosos se le ha llamado de diferentes maneras: ambiente, atmósfera, clima organizacional o satisfacción en el trabajo, entre otros.

Sobre este aspecto, Kahr (2010), reconoce ese ambiente laboral como clima institucional definiéndolo como el ambiente que se genera a partir de las vivencias cotidianas de los miembros de la organización $\mathrm{y}$ que tiene que ver con las actitudes, creencias, valores y motivaciones que tiene cada trabajador y se expresa en las relaciones personales y profesionales

El concepto de clima institucional es general y se refiere al ambiente global de la organización. En esta investigación se pretendió estudiar el clima institucional referido a lo administrativo, es decir, el clima que surge alrededor de las actividades administrativas y de interacción social en las organizaciones objeto de estudio.

Al respecto, Morán de Volkwein $(1992$ c.p, Santana y Araujo 2007), define el clima institucional desde cuatro perspectivas: en lo estructural, lo perceptual, la interactiva y la cultural.

Bajo el enfoque estructural, estos autores exponen que el clima es considerado como una manifestación de la estructura de la organización, independientemente de las percepciones de los individuos. El clima se forma porque los miembros de la organización se encuentran expuestos a unas mismas características estructurales.

Desde la perspectiva perceptual, la base de la formación del clima organizacional entra dentro del individuo. En este sentido, el clima es un proceso psicológico que describe las condiciones de la organización (Joyce y Slocum, 1982; Schneider y Reichers, 1983).

En relación con la perspectiva interactiva, la interacción de los individuos marca las respuestas dadas a las situaciones objetivas y compartidas por la organización en la base para la formación del clima organizacional (Poole y McPhee, 1983; Jackofsky y Slocum, 1988).

Finalmente, el enfoque cultural hace mención al clima que crea un grupo de interacciones individuales que comparte un marco común de referencia, como es la cultura organizacional (Berger y Luckman, 1967).

Estás últimas perspectivas no se trataron en la delimitación conceptual del clima puesto que se solapaban en el concepto de cultura organizacional. Es por ello que en esta investigación se estudió el clima institucional bajo los aspectos trabajados por Moran y Volkwein (1992 c.p. Santana y Araujo, 2007), como enfoque estructural, Rodríguez y otros (2010) y adecuado según Hurtado (2015), en un instrumento bajo la dimensión de clima institucional administrativo.

En este instrumento se reconocieron como sinergias del clima institucional administrativo a: la dirección, formación del talento humano, flujo de información, apertura al cambio y solución de conflictos 
MÉTODO

La investigación Clima institucional administrativo de la organización educativa Escuela de Educación de Colombia, se ubicó en el estudio descriptivo, siendo la variable independiente el clima institucional administrativo. El diseño de la investigación fue considerado de campo transeccional contemporáneo unieventual.

Para la recolección de datos la técnica utilizada fue la encuesta y el instrumento un cuestionario denominado Escala de Clima Institucional Administrativo (CIA), el cual constaba de veinticuatro (24) items con cinco alternativas de respuestas, su validez se calculó mediante el juicio de expertos y una confiabilidad de 0,97 de Alfa de Cronbach mediante el programa SPSS 18. En el análisis de los resultados se utilizó la estadística descriptiva, específicamente la frecuencia absoluta y porcentual, y la mediana como medida de tendencia central por cuanto la variable se midió en un nivel ordinal.

La población estuvo conformada por 19 empleados de la organización educativa Escuela de Educación de Colombia.

\section{RESULTADOS Y DISCUSIÓN}

\section{Análisis global del evento clima institucional administrativo}

Con relación a la descripción del clima institucional administrativo de la organización Educativa Esesco, los resultados que se presentan en el cuadro 1 reflejaron una mediana de 5,83 puntos sobre 20 puntos, que al ser comparado con el baremo de interpretación indicó que existía en la institución estudiada un clima institucional administrativo desfavorable.

Estos resultados indicaron que el clima institucional administrativo de la organización educativa Escuela de Educación de Colombia proporciona un ambiente poco agradable a partir de las vivencias cotidianas de sus miembros, donde las actitudes, creencias, valores y motivaciones que tenía cada trabajador se expresaba en las relaciones personales y profesionales.

En el gráfico 1 se observó una distribución estadística que dividió el recorrido de los valores alcanzados en cuatro (4) partes; cada una de éstas contenía el $25 \%$ del total de los resultados, siendo la mediana el valor central (50\%) que dividía la distribución. En cada cuartil se ubicó la misma cantidad de elementos o sujetos, es decir, los cuartiles representaban la homogeneidad o heterogeneidad de los puntajes. De esta manera se observó en la gráfica del clima institucional administrativo una distribución simétrica, el grupo era homogéneo, es decir, presentaba características iguales en cuanto a la percepción que tenían del clima en la organización estudiada.

Cuadro 1. Baremo de interpretación de la escala Clima Institucional Administrativo

\begin{tabular}{cc}
\hline Escala & Interpretación \\
\hline $0-3,99$ & Muy desfavorable \\
$4-7,99$ & Desfavorable \\
$8-11,99$ & Medianamente favorable \\
$12-15,99$ & Favorable \\
$16-20$ & Muy favorable \\
\hline
\end{tabular}


Cuadro 2. Estadísticos del Clima institucional administrativo

\begin{tabular}{lcr}
\hline $\mathbf{N}$ & Válidos & $\mathbf{1 9}$ \\
& Perdidos & $\mathbf{0}$ \\
\hline Mediana & & 5,83 \\
Mínimo & & 1,67 \\
Máximo & & 20,00 \\
Percentiles & 25 & 4,37 \\
& 50 & 5,83 \\
& 75 & 6,66 \\
\hline
\end{tabular}

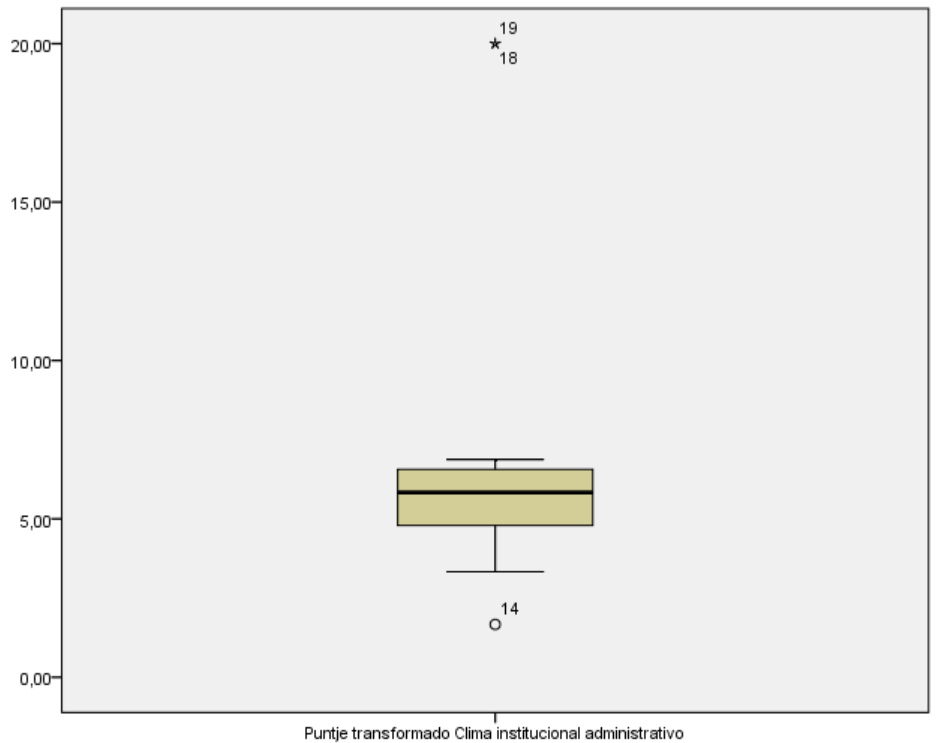

Gráfico 1. Caja bigote del Clima institucional administrativo

Sin embargo, el grupo que estaba por debajo de la mediana obtuvo puntajes más variados, lo cual indicaba que era más heterogéneo que el que estaba por encima de la mediana. Se presentaron tres casos atípicos. El caso 14 con un puntaje de 1,67 donde se describía a un empleado sin formación en educación como docente. Los casos 18 y 19 con puntajes 20 por encima del grupo eran el gerente general y el gerente administrativo de la organización.

Cuadro 3. Categorías de Clima institucional administrativo

\begin{tabular}{lcccc}
\hline & Frecuencia & Porcentaje & $\begin{array}{c}\text { Porcentaje } \\
\text { válido }\end{array}$ & $\begin{array}{c}\text { Porcentaje } \\
\text { acumulado }\end{array}$ \\
\hline Muy desfavorable & 3 & 15,8 & 15,8 & 15,8 \\
Desfavorable & 14 & 73,7 & 73,7 & 89,5 \\
Muy favorable & 2 & 10,5 & 10,5 & 100,0 \\
\hline Total & $\mathbf{1 9}$ & $\mathbf{1 0 0 , 0}$ & $\mathbf{1 0 0 , 0}$ & \\
\hline
\end{tabular}


Con relación a la distribución de las unidades de estudio en las categorías (ver cuadro 3), se observó que un $73,7 \%$ de la población opinaba que en la organización existía un clima institucional administrativo desfavorable, mientras un $15,8 \%$ creía que era muy desfavorable y solo un $10,5 \%$ opinó que era favorable.

\section{Análisis de las sinergias de clima institucional administrativo}

En el cuadro 4, se observaron los resultados de las dimensiones del clima institucional administrativo (dirección, formación del talento humano, condiciones de trabajo adecuadas, flujo de información, involucración con el cambio, solución de conflicto), los cuales reflejaban que todas las dimensiones se ubicaban en la categoría de desfavorable con medianas de $5 ; 5 ; 6,25 ; 6,67$; 5; 6,25 puntos de un máximo de 20 puntos.

No obstante, la dimensiones que presentaron menores puntajes en sus medianas fueron la dirección, formación del talento humano e involucración para el cambio, lo cual indicó que los empleados opinaban que existían grandes desviaciones en la forma como se daban las instrucciones de las actividades, la capacitación del personal y el manejo del cambio en los procesos administrativos de la empresa.

Cuadro 4. Estadísticos de las sinergias de Clima institucional administrativo

\begin{tabular}{cccccccc}
\hline & & $\begin{array}{c}\text { Puntaje } \\
\text { transformado } \\
\text { Dirección }\end{array}$ & $\begin{array}{c}\text { Puntaje } \\
\text { transformado } \\
\text { formación del } \\
\text { talento } \\
\text { humano }\end{array}$ & $\begin{array}{c}\text { Puntaje } \\
\text { transformado } \\
\text { condiciones de } \\
\text { trabajo } \\
\text { adecuado }\end{array}$ & $\begin{array}{c}\text { Puntaje } \\
\text { transformado } \\
\text { flujo de } \\
\text { información }\end{array}$ & $\begin{array}{c}\text { Puntaje } \\
\text { transformado } \\
\text { involucración } \\
\text { con el cambio }\end{array}$ & $\begin{array}{c}\text { Puntaje } \\
\text { transformado } \\
\text { solución de } \\
\text { conflictos }\end{array}$ \\
\hline $\mathrm{N}$ & $\begin{array}{c}\text { Validos } \\
\text { Perdido }\end{array}$ & 19 & 19 & 19 & 19 & 19 & 19 \\
& $\mathrm{~S}$ & 0 & 0 & 0 & 0 & 0 & 0 \\
Mediana & & 5.00 & 5.00 & 6.25 & 6.67 & -5.00 & 6.25 \\
Mínimo & & 2 & 0 & 1 & 2 & 0 & 1 \\
Máximo & & 20 & 20 & 20 & 20 & 20 & 20 \\
Percentiles & 25 & 5.00 & 3.75 & 3.75 & 5.00 & 3.75 & 3.75 \\
& 50 & 5.00 & 5.00 & 6.25 & 6.67 & 5.00 & 6.25 \\
& 75 & 7.00 & 6.25 & 7.50 & 8.33 & 7.50 & 8.75 \\
\hline
\end{tabular}

Pudo apreciarse que la dimensión con mejor puntuación fue la de flujo de información con una mediana de 6,67 de un máximo de 20 puntos.

Según la distribución de las dimensiones del clima institucional administrativo con cajas alargadas, el gráfico 2 indicó una distribución asimétrica con un grupo heterogéneo, es decir, presentaba características dispersas en cuanto al clima social escolar. Se observaron casos atípicos con puntaje muy bajos al grupo y otro con puntajes que sobresalían al grupo. 


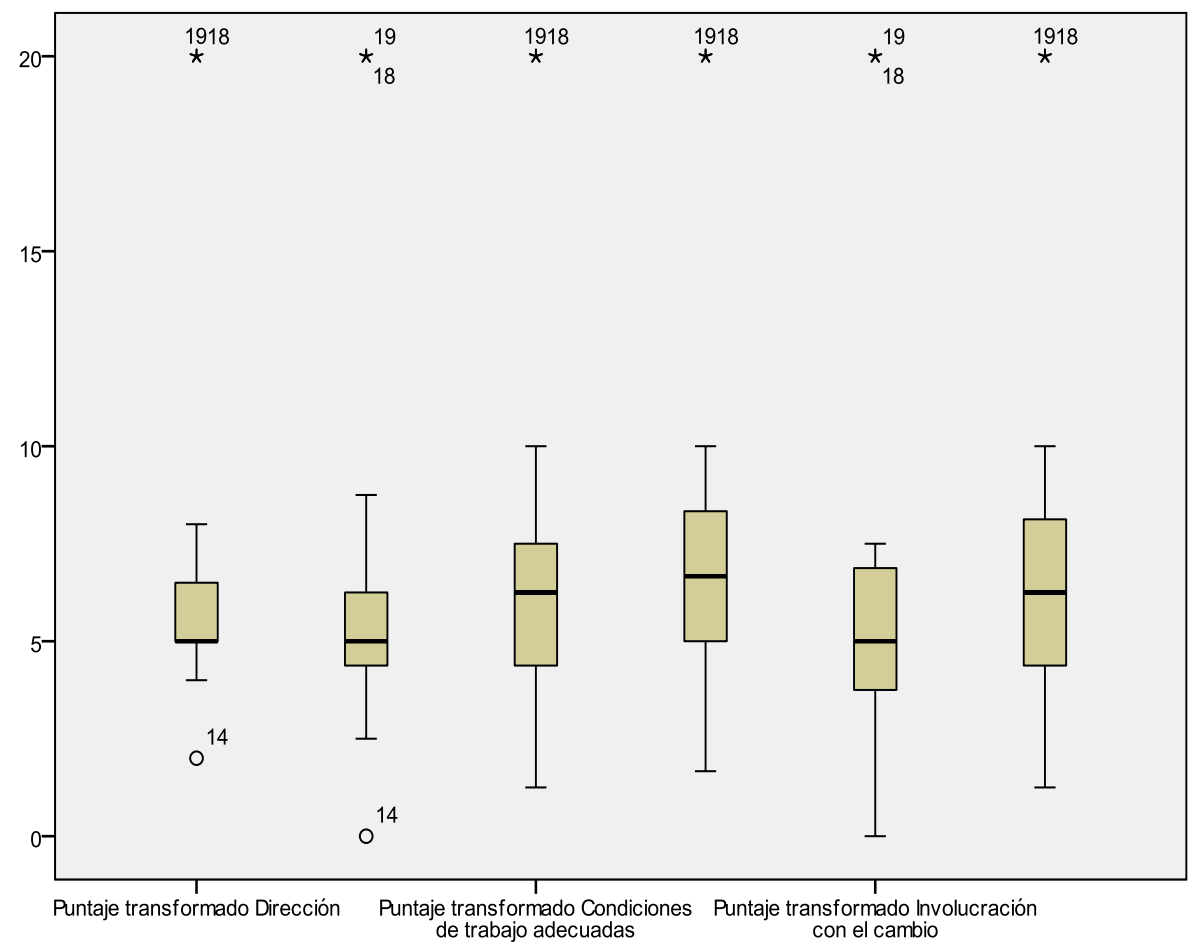

Gráfico 2. Caja bigote de las sinergias de clima institucional administrativo

\section{Análisis de la sinergia dirección}

Con respecto a la sinergia dirección, los resultados del cuadro 4 reflejaron una mediana de 5 puntos sobre 20, la cual se ubicaba en el baremo de interpretación en la categoría de desfavorable. Esto indicó que los empleados de la organización educativa Escuela de Educación de Colombia opinaban que la organización no proporcionaba una orientación clara a las actividades a realizar y metas institucionales. De igual manera, las responsabilidades de cada subalterno y las interacciones con otras personas y grupos no estaban establecidas.
En el gráfico 2 se observó una caja bigote con una distribución asimétrica, donde el $50 \%$ del grupo y el $25 \%$ tenían los mismos puntajes, por lo que el grupo era heterogéneo. El puntaje mínimo era de 2 puntos y el máximo de 20 puntos. Se observaron tres casos atípicos.

El caso 14 con un puntaje de 2 puntos correspondía a la persona con profesión de regente de farmacia quien se desempeñaba en la organización como docente.

El caso 18 y 19 con 20 puntos, representaban la coordinación general y al director general de la organización, quienes eran personas con formación gerencial. 
Cuadro 5. Categorías de la sinergia Dirección

\begin{tabular}{lcccc}
\hline & Frecuencia & Porcentaje & $\begin{array}{c}\text { Porcentaje } \\
\text { válido }\end{array}$ & $\begin{array}{c}\text { Porcentaje } \\
\text { acumulado }\end{array}$ \\
\hline Muy desfavorable & 1 & 5,3 & 5,3 & 5,3 \\
Desfavorable & 14 & 73,7 & 73,7 & 78,9 \\
Medianamente favorable & 2 & 10,5 & 10,5 & 89,5 \\
Muy favorable & 2 & 10,5 & 10,5 & 100,0 \\
\hline Total & $\mathbf{1 9}$ & $\mathbf{1 0 0 , 0}$ & $\mathbf{1 0 0 , 0}$ & \\
\hline
\end{tabular}

Con respecto a la distribución de la población en las categorías de la sinergia dirección, se observó en el cuadro 5 que el mayor porcentaje de la unidad de estudio se ubicó en desfavorable $(73,75)$, seguido de un $10,5 \%$ para las categorías de medianamente favorable y muy favorable respectivamente y un $5,3 \%$ en muy desfavorable.

\section{Análisis de la sinergia formación del talento humano}

En lo que corresponde a esta sinergia, los resultados obtenidos en el cuadro 4 , con un valor de la mediana de 5 puntos de un máximo de 20 ubicaron al grupo en la categoría de desfavorable. Esto reflejó que la población encuestada creía que en Esesco no existía un clima administrativo que favoreciera la formación y el desarrollo de competencias de los empleados.

En el gráfico 2 se observó una distribución asimétrica heterogénea, es decir, el grupo poseía características dispersas en cuanto a la percepción del clima institucional administrativo, pero el grupo que estaba por debajo de la mediana era más homogéneo. Los mismos casos atípicos se presentaron en la sinergia dirección. En esta sinergia el caso 14 obtuvo 0 puntos de un máximo de 20 .

En el cuadro 6, se presentó la distribución de la unidad de estudio en las categorías de la sinergia, donde se observó que un $57,9 \%$ del grupo considero desfavorable la formación del talento humano, un $26,3 \%$ muy desfavorable mientras un $10,5 \%$ se ubicó en muy favorable y $5.3 \%$ medianamente favorable.

Cuadro 6. Categorías de Formación del talento humano

\begin{tabular}{lcccc}
\hline & Frecuencia & Porcentaje & $\begin{array}{c}\text { Porcentaje } \\
\text { válido }\end{array}$ & $\begin{array}{c}\text { Porcentaje } \\
\text { acumulado }\end{array}$ \\
\hline Muy desfavorable & 5 & 26,3 & 26,3 & 26,3 \\
Desfavorable & 11 & 57,9 & 57,9 & 84,2 \\
Medianamente favorable & 1 & 5,3 & 5,3 & 89,5 \\
Muy favorable & 2 & 10,5 & 10,5 & 100,0 \\
\hline Total & $\mathbf{1 9}$ & $\mathbf{1 0 0 , 0}$ & $\mathbf{1 0 0 , 0}$ & \\
\hline
\end{tabular}

Análisis de la sinergia condiciones de trabajo adecuadas

Con respecto a la sinergia condiciones de trabajo adecuadas los resultados del cuadro 4, reflejaron una mediana de 6,25 puntos de un máximo de 20, la cual se ubicó en la categoría de desfavorable en el baremo de interpretación. Estos resultados reflejaron que los empleados de la organización educativa Escuela de Educación de Colombia opinaban que en la empresa las condiciones de trabajo no daban respuesta a sus expectativas, es decir, los espacios, recursos, 
tecnología y condiciones ambientales existentes no eran óptimas para la ejecución de las actividades administrativas.

En el gráfico 2 se observó una distribución asimétrica con un grupo con características heterogéneas, es decir, con puntajes diversos. Un puntaje mínimo de 1 punto y el máximo de 20. El cuartil 75 se ubicó en 7,5, lo cual indicaba que el 75\% de la población encuestada creía que el ambiente laboral tenía solo un $37 \%$ de las condiciones adecuadas de trabajo.
Los casos atípicos fueron los mismos que en la sinergia dirección y formación del talento humano.

La distribución de la población en las categorías de la sinergia condiciones de trabajo adecuadas (cuadro 7), expresaron que un $52,6 \%$ de la población se agrupó en las categorías desfavorables y muy desfavorables, mientras que un $26,3 \%$ en mediana-mente favorable y favorable un $10,5 \%$.

Cuadro 7. Categoría de Condiciones de trabajo adecuadas

\begin{tabular}{lcccc}
\hline & Frecuencia & Porcentaje & $\begin{array}{c}\text { Porcentaje } \\
\text { válido }\end{array}$ & $\begin{array}{c}\text { Porcentaje } \\
\text { acumulado }\end{array}$ \\
\hline Muy desfavorable & 5 & 26,3 & 26,3 & 26,3 \\
Desfavorable & 10 & 52,6 & 52,6 & 78,9 \\
Medianamente favorable & 2 & 10,5 & 10,5 & 89,5 \\
Muy favorable & 2 & 10,5 & 10,5 & 100,0 \\
\hline Total & $\mathbf{1 9}$ & $\mathbf{1 0 0 , 0}$ & $\mathbf{1 0 0 , 0}$ & \\
\hline
\end{tabular}

Análisis de la sinergia flujo de información

Los resultados del cuadro 4 reflejaron una mediana de 6,67 puntos de un máximo de 20 puntos para la sinergia flujo de información, lo cual indicaba que eran desfavorable los canales de comunicación y el flujo de información en la organización estudiada.

En el gráfico 2 se observó una distribución asimétrica, donde el grupo tenía características dispersas en sus puntajes por lo que era heterogéneo. Los casos atípicos coincidieron con los de las sinergias antes estudiadas.

Con respecto a la distribución de la población en las categorías de la sinergia flujo de información (cuadro 8), se percibió que el $47,4 \%$ opinó que el flujo de información era desfavorable, el 26,3 lo consideró medianamente favorable, el 15,8\% muy desfavorable y solo el 10,5 creía que es muy favorable.

Cuadro 8. Categorías de Flujo de información

\begin{tabular}{lcccc}
\hline & Frecuencia & Porcentaje & $\begin{array}{c}\text { Porcentaje } \\
\text { válido }\end{array}$ & $\begin{array}{c}\text { Porcentaje } \\
\text { acumulado }\end{array}$ \\
\hline Muy desfavorable & 3 & 15,8 & 15,8 & 15,8 \\
Desfavorable & 9 & 47,4 & 47,4 & 63,2 \\
Medianamente favorable & 5 & 26,3 & 26,3 & 89,5 \\
Muy favorable & 2 & 10,5 & 10,5 & 100,0 \\
\hline Total & $\mathbf{1 9}$ & $\mathbf{1 0 0 , 0}$ & $\mathbf{1 0 0 , 0}$ & \\
\hline
\end{tabular}




\section{Análisis de la sinergia apertura al cambio}

Con respecto a esta sinergia, el cuadro 4 mostró que la mediana obtenida fue de 5 puntos de un máximo de 20 puntos, lo cual se ubicó en la categoría de desfavorable según el baremo de interpretación, en razón de este resultado generado por los empleados encuestados pudo inferirse que en la organización los procesos de cambio se viven de manera improvisada, lo cual indicaba que la institución no estaba abierta a los cambios que impone el entorno administrativo en una institución educativa.
Por su parte, en el gráfico 2 se observó una distribución asimétrica, donde el grupo era heterogéneo; sin embargo, el grupo por encima de la mediana era más homogéneo que el que estaba por debajo. Los valores atípicos estaban representados en los mismos casos que en las demás sinergias.

Con respecto a la distribución de la población en las categorías de la sinergia apertura al cambio (cuadro 9) se percibió que un 57,9\% opinó que el flujo de información era desfavorable, un $31,6 \%$ que era muy desfavorable, en tanto un $10,5 \%$ creía que era muy favorable.

Cuadro 9. Categorías de apertura al cambio

\begin{tabular}{lcccc}
\hline & Frecuencia & Porcentaje & Porcentaje válido & $\begin{array}{c}\text { Porcentaje } \\
\text { acumulado }\end{array}$ \\
\hline Muy desfavorable & 6 & 31,6 & 31,6 & 31,6 \\
Desfavorable & 11 & 57,9 & 57,9 & 89,5 \\
Muy favorable & 2 & 10,5 & 10,5 & 100,0 \\
\hline Total & $\mathbf{1 9}$ & $\mathbf{1 0 0 , 0}$ & $\mathbf{1 0 0 , 0}$ & \\
\hline
\end{tabular}

Análisis de la sinergia solución de conflictos

$\mathrm{Al}$ analizar la sinergia solución de conflictos, el cuadro 4 mostró una mediana de 6,25 lo que indicó que los encuestados tenían una opinión desfavorable sobre la forma como en la organización educativa Escuela de Educación de Colombia se manejaba la solución de conflicto. Esta sinergia es muy importante en el clima administrativo de una institución, por cuanto los conflictos son propios de los ambientes de interacción personal, sin embargo, es necesario que los directivos o gerentes de las organizaciones utilicen la mediación en la solución de diferencias a fin de propiciar acuerdos entre las partes.

Igualmente, el gráfico 2 reflejó una distribución asimétrica, con un grupo con características dispersas, por lo que se consideraba heterogéneo. Los casos atípicos eran los mismos que en las sinergias anteriores.

La distribución de la población estudiada en las categorías de la sinergia solución de conflicto que se observó en el cuadro 10, reflejaba que un $47,4 \%$ se ubicó en desfavorable, un $26,3 \%$ en muy desfavorable, mientras el $15,8 \%$ se ubicó en medianamente favorable y solo un 10,5 en muy favorable. 
Cuadro 10. Categorías de Solución de conflicto

\begin{tabular}{lcccc}
\hline & Frecuencia & Porcentaje & $\begin{array}{c}\text { Porcentaje } \\
\text { válido }\end{array}$ & $\begin{array}{c}\text { Porcentaje } \\
\text { acumulado }\end{array}$ \\
\hline Muy desfavorable & 5 & 26,3 & 26,3 & 26,3 \\
Desfavorable & 9 & 47,4 & 47,4 & 73,7 \\
Medianamente favorable & 3 & 15,8 & 15,8 & 89,5 \\
Muy favorable & 2 & 10,5 & 10,5 & 100,0 \\
\hline Total & $\mathbf{1 9}$ & $\mathbf{1 0 0 , 0}$ & $\mathbf{1 0 0 , 0}$ & \\
\hline
\end{tabular}

\section{Discusión}

Los resultados obtenidos indicaron que el clima institucional administrativo de la organización educativa Escuela de Educación de Colombia era desfavorable, en virtud de que logró una mediana de 5,83 puntos de un máximo de 20. Esto reflejaba que los gerentes de la institución no se preocupaban por crear un ambiente laboral donde las funciones estuviesen claramente definidas, con un personal capacitado para realizar sus actividades dentro de los requisitos exigidos para el éxito de la institución.

Es de notarse que estos resultados eran claramente contrarios a los planteamientos de Hurtado (2015), cuando manifiesta que clima institucional administrativo es el ambiente producto de la influencia que ejercen los directivos en las actividades laborales a través de pautas, normas, objetivos y lineamientos para lograr eficiencia y eficacia, por medio de la habilidad de orientar, mediar, dirigir y comunicar oportunamente.

Además, la gerencia debía involucrar al personal que cumplía funciones administrativas en los procesos importantes de la gerencia, es decir, desarrollar una acción orientadora dirigida a los procesos direccionales, mediante el cual el personal se sintiera motivado y tomado en cuenta para el crecimiento de la organización.

De igual forma, la organización educativa Escuela de Educación de Colombia debería propiciar condiciones de trabajo adecuadas que vayan más allá de las condiciones físicas de la institución, con un flujo de comunicación dirigido en todas las direcciones, sin interferencias, con la finalidad de que el personal se sienta informado e involucrado en los procesos que se ejecutan en la corporación.

Así mismo, los gerentes de la organización educativa Escuela de Educación de Colombia, deberían abrir espacios que explique los cambios a los que se enfrenta la organización con la finalidad de que sus empleados cognitivamente los procesen $\mathrm{y}$ participen activamente en los mismos al considerarse los principales partícipes.

Con relación a los resultados de las sinergias que conforman el clima institucional administrativo de la organización educativa Escuela de Educación de Colombia se observó que todas se ubicaron en una categoría de desfavorable con medianas que oscilaron ente 5 puntos y un máximo de 6,67 puntos de un máximo de 20 puntos.

Al estudiar el comportamiento de la sinergia dirección se consideró desfavorable ya que logró una mediana de 5,83 puntos de un máximo de 20, lo que denotó que en la organización educativa Escuela de Educación de Colombia existían desviaciones en cuanto a que los directores poco se preocupaban de que el personal conociera sus funciones $y$ entendiera el trabajo que debía realizar en la institución; además, algunas veces la solución de los problemas de algunos proyectos era planteada con poca claridad. Del mismo modo, eran irrisorios los criterios que utilizaba la gerencia para evaluar el trabajo desempeñado y las actividades a realizar eran 
descritas de forma confusa.

Estos resultados están en discrepancias con los postulados teóricos de Rodríguez y otros (2010), cuando expresan que la dirección proporciona el sentido de orientación de las actividades de una unidad de trabajo, pues establece los objetivos e identifica claramente las metas que se deben alcanzar y los medios para lograrlo.

En referencia a la sinergia formación de talento humano, la mediana fue de 5 puntos de un máximo de 20, ubicándola en una categoría de desfavorable, esto implicaba que en la organización educativa Escuela de Educación de Colombia, las personas que laboraban carecían de oportunidades de capacitación y actualización en el manejo de las tecnologías de la información y comunicación herramienta importante $\mathrm{y}$ necesaria para el cumplimiento de las actividades administrativas en la organización, además los empleados poco participaban en las actividades de actualización para el logro de un mejor desempeño, así como se evidenció ausencia de información por parte de los directivos sobre las nuevas técnicas administrativas.

Los resultados obtenidos estaban en divergencia con la postura de teóricos expertos como Alles (2006), cuando manifiesta que la formación del talento humano cumple con la necesidad de adoptar y diseñar acciones que le permitan estar dentro de un sistema planificado y acorde con el ambiente que le rodea para mantenerse en una posición proactiva y de innovación ante el futuro.

En cuanto a la sinergia referida a las condiciones de trabajo en la organización educativa Escuela de Educación de Colombia, esta logró una mediana de 6,25 puntos de un máximo de 20, lo que la ubicó en la categoría desfavorable. Esta valoración reflejó que la institución a pesar de contar con una infraestructura apropiada, con recursos tecnológicos adecuados y todos los elementos necesarios para ejecutar un buen trabajo, el ambiente físico era inapropiado, con malas relaciones interpersonales que ocasionaban situaciones estresantes que redundaban en la ejecución del trabajo, además, los gerentes poco se vinculaban con el personal para que en conjunto desarrollen una labor más productivo y propicien en la institución un ambiente agradable para ejecutar el trabajo.

Estos resultados difieren de la postura de Castillo y Prieto (1990), cuando dicen que las condiciones de trabajo son todo aquello que gira en torno al trabajo desde la perspectiva de cómo repercute el trabajo en las personas. Por ello, las condiciones de trabajo no sólo son la higiene, seguridad, los aspectos físicos, sino también determinan estas condiciones los aspectos psíquicos.

También Rodríguez y otros (2010), expresan que las condiciones ambientales físicas y psicosociales en las que se realiza el trabajo, así como la calidad y cantidad de los recursos que se suministran para el cumplimiento de las funciones asignadas, deben ser congruentes con la naturaleza misma del trabajo. Cuando la adecuación de estas condiciones no se da, se produce insatisfacción en el trabajador, lo cual repercute en bajos niveles de desempeño y en la calidad de los servicios producidos.

Estos planteamientos permiten inferir que la organización educativa Escuela de Educación de Colombia está obligada a generar climas de trabajo acordes con las expectativas de sus empleados, por lo cual se requiere que los gerentes sean expertos $\mathrm{y}$ conscientes de la importancia que tienen unas condiciones de trabajo óptimas para la eficiencia y eficacia de una organización.

Para la sinergia donde se midió el flujo de información en la organización educativa Escuela de Educación de Colombia, los resultados se ubicaron en la alternativa 
desfavorable por alcanzar una mediana 6,67 puntos de un máximo de 20, lo que indicó que las personas que laboraban en la organización, estaban carentes de información sobre los nuevos proyectos que la empresa planeaba ejecutar, sobre las pautas como debían desarrollar las actividades, así como la poca divulgación de información sobre los procesos a desarrollar.

Estos resultados se encuentran en discrepancias con la postura de Contreras (2012), cuando explica que el buen flujo de comunicación contribuye en gran parte con la forma de pensar a nivel interno y externo de la organización. Sin duda un flujo comunicacional adecuado genera cambios en el comportamiento humano, actitudes positivas, decisiones acertadas, soluciones de proble-mas y trabajo colaborativo, entre los empleados.

Así mismo, Rodríguez y otros (2010) expresan que, para obtener la acción coordinada entre personas y grupos, es necesario comunicarse e intercambiar información sobre los propósitos comunes y los medios que cada uno posee para contribuir al logro de ellos.

Ante estos argumentos se hace necesario que los directivos de la organización educativa Escuela de Educación de Colombia estén conscientes de una serie de elementos a la hora de iniciar un proceso de comunicación, de tal manera que la comunicación influya en la percepción, la personalidad, las emociones de cada trabajador, lo cual conlleva a un clima institucional favorable $\mathrm{y}$ con empleados dispuestos a aprender y a comprometerse con la institución.

En referencia a la sinergia apertura al cambio, los resultados se ubicaron en la alternativa desfavorable, con una mediana de 5 puntos de un máximo de 20, infiriendo a través de esta valoración que la corporación poco ha ofrecido a sus empleados una sensibilización en el manejo del cambio, ante lo cual se observa un rechazo por parte de los trabajadores al uso de tecnologías actualizadas, además, brindaba escaso respaldo a las iniciativas de trabajo de los grupos. De igual manera, los directores poco propiciaban cambios en las actividades a realizar, lo que había llevado a la desmotivación del personal para desarrollar cambios favorables en las actividades a realizar.

Estos resultados están en disparidad con la posición de expertos como Conger (2000), cuando expresa que para lograr el cambio es necesario tener claro que el personal necesita tiempo para ajustarse, comprender y comprometerse con el cambio, por ello reviste gran importancia la comunicación en ambos sentidos e involucrar al personal para que aporte soluciones, estando consciente que algunas veces el proceso de cambio lleva consigo la negociación, sobre todo con aquellas personas que se oponen a él para el beneficios de ambas partes.

De la misma forma, Rodríguez y otros (2010) expresan que las organizaciones están constantemente dinamizadas por múltiples procesos de cambio, profundos y acelerados, que permanentemente plantean al hombre nuevas interrogantes. Ante esta realidad el hombre no permanece impasible; siempre adopta una postura. La actitud comprometida, orientando y promoviendo el cambio, mediante la construcción del futuro a partir de la experiencia asimilada en el pasado, es la verdadera respuesta ante el proceso de cambio.

Al estudiar la sinergia solución de conflicto, los resultados se ubicaron en la categoría desfavorable, con una mediana de 6,25 puntos de un máximo de 20, esto indicaba que los gerentes de la empresa muy poca veces planteaban discusiones constructivas de los problemas laborales, además, había escaso interés en la solución de 
Los mismos, lo que ocasionaba que los empleados muchas veces estuviesen inmersos en un ambiente de trabajo hostil o poco armonioso.

Estos resultados se muestran contrarios a la postura de González (2006), cuando plantea que en la consecución de un buen clima personal y laboral es importante el manejo de los conflictos. Para dicho autor la solución al conflicto, en gran parte, descansa en la dirección y la mejor oportunidad de solución se consigue en la primera etapa de la mediación, es decir, antes que el conflicto se agrave. De igual manera considera que la ausencia de conflicto en una organización no es aconsejable, ya que a través de estos se propicia un reequilibrio de los diversos intereses organizacionales.

Por su parte, Rodríguez y otros (2010), expresan que el conflicto se considera como parte inherente en la vida institucional. En las organizaciones siempre surgen conflictos que se originan por diversas causas, aunque en definitiva tienen un trasfondo común: son diferencias de percepciones e intereses que compiten sobre una misma realidad.

\section{CONCLUSIONES}

Con respecto a la descripción del clima institucional administrativo de la organización educativa Escuela de Educación de Colombia, los resultados obtenidos indicaron que en la institución estudiada existía un clima institucional administrativo desfavorable, es decir, en la organización educativa Escuela de Educación de Colombia, el clima institucional administrativo poco proporcionaba un ambiente agradable a partir de las vivencias cotidianas de sus miembros, donde las actitudes, creencias, valores y motivaciones que tiene cada trabajador y se expresa en las relaciones personales y profesionales.

Con relación al proceso de dirección en la organización educativa Escuela de Educación de Colombia, los resultados se ubicaron en la categoría desfavorable; esto indicaba que los empleados de la organización no poseían una orientación clara a las actividades a realizar y metas institucionales, de igual manera las responsabilidades de cada subalterno y las interacciones con otras personas y grupos no estaban establecidas.

Al caracterizar la sinergia formación del talento humano en la organización educativa Escuela de Educación de Colombia, los resultados arrojados la ubicaron en la categoría de desfavorable; esto reflejaba que la población encuestada percibía que en la organización no existía un clima administrativo que favorezca la formación y el desarrollo de competencias de los empleados.

De igual manera, los resultados de la sinergia condiciones de trabajo en la organización educativa Escuela de Educación de Colombia, la ubicaron en la categoría de desfavorable, lo que podría manifestarse en los empleados en problemas de salud al no brindarles la organización bienestar físico, mental y social. Así como en poca motivación al desarrollar sus actividades pues las condiciones de trabajo a nivel social no satisfacen las necesidades y expectativas de los empleados. Igual situación se hace presenta con respecto a la sinergia flujo de información en la organización, los resultados la ubicaron en la categoría desfavorable ya que los canales de comunicación y el flujo de información en la organización estudiada eran inapropiados.

Los resultados de las sinergias proceso de apertura al cambio y solución de conflictos en la organización nuevamente se ubicaron en la categoría desfavorable, lo cual se evidenciaba en la resistencia del personal administrativo de la organización a asumir las innovaciones y transformaciones del proceso educativo, así como en el poco involucramiento de los aspectos emocionales y sentimientos de los trabajadores en diálogos 
constructivos en situaciones de conflictos.

\section{REFERENCIAS}

Alles, M. (2006). Desarrollo del talento humano: basado en competencias. Argentina: Granica S.A.

Alvares, F. (2017). El clima institucional como factor determinante en el rendimiento académico de los alumnos de la institución educativa Técnico Industrial Simona Duque del municipio de marinilla. Recuperado en: http://bibliotecadigital.udea.edu.co/bitst ream/10495/9171/1/AlvarezFrancisco_ 2017_ClimaRendimientoAcademcico.pdf. (2 de septiembre de 2018)

Balza, A. (2010). Educación, investigación y aprendizaje. Una hermaneusis desde el pensamiento complejo y transdisciplinarios. $2^{\circ}$ edición. Caracas. Venezuela: Fondo editorial APUNESR

Berger, L. y Luckmann, T. (1967). The social construction of reality; A treatise in the sociology of knowledge. Garden City, NY: Doubleday

Brunet, L. (2005). El clima de trabajo en las organizaciones. 6o edición. México: Trillas

Campbell y otros (1970). Managerial behavior, performance, a effectiveness. New York: Mc Graw Hill

Castillo, J. y Prieto, C. (1990): Condiciones de trabajo, un enfoque renovador de la sociología del trabajo, Madrid: CIS

Conger, A. (2000). El manual del cambio para líderes. México: Paidos.

Contreras, H. (2012). RRPP Net Porat. Recuperado en: http:/rrppnet.com.ar/comorganizacional. htm. (13 de mayo de 2018)

Davis, K. y Newtrom, J. (1999). Comportamiento Humano en el Trabajo. México: Mac Graw Hill Interamericana

Gento, S. (1994). Instituciones educativas para la calidad total. México: La Muralla.

Goncalves, A. (2001). Fundamentos del clima organizacional. México: Editorial Sociedad Latinoamericana para la Sociedad

González, M. (2006) Gestión de Conflictos Laborales. $1^{\circ}$ edición. España: Innova.

Hamel, G. y Breen, B. (2008). El Futuro de la
Administración. Colombia: Norma.

Hurtado, J. (2015). Clima científico institucional y producción científica en las universidades: Hacia el desarrollo de una teoría. Tesis doctoral, ILU Willemstad, Curaçao

Jackofsky, F. y Slocum, W. (1988). A longitudinal study of climate. Journal of Organizational Behavior, 9, 319-334

Joyce, F. y Slocum, W. (1982). Climate discrepancy: Redefining the concepts de psychological and organitational climate. Human r Relations, 35, 11, p. 951-852

Kahr, M. (2010). Clima institucional escolar y gestión directiva. Trabajo de grado. Universidad Abierta Interamericana. Buenos Aires. Argentina

Lanz, R. (2004). Universidad y pensamiento postmoderno. Hay chance para la razón estética. En revista Relea. № 9. Cipost UCV. Caracas. Venezuela

Litwin, G. y Taguiri, R. (1969). Organizational climate: Explorations of a concept. Boston: Harvard Business School, Division of Research

Litwin, H. y Stringer, A. (1968). Motivation and organizational climate. Boston: Harvard Business School Press

Molocho, N. (2010). Influencia del clima organizacional en la gestión institucional de la sede administrativa UGEL № 01Lima Sur-2009. Recuperado en: http://cybertesis.unmsm.edu.pe/handle/ cybertesis/3230. (26 de agosto de 2018)

Poole, S. y McPhee, D. (1983). A structurational analysis of ognanitational climate: En Putnam y Pacanowsky: Communication and organitations: Ad interpretative approach. Beverly Hills: Sage P. 195-219

Rodríguez, A; Álvarez, A; Sosa, I; De Vos, P; Bonet, M; Van der Stuyft, P. (2010). Inventario del clima institucional como una herramienta necesaria para evaluar la calidad del trabajo. Revista Cubana de Higiene y Epidemiología. 48(2) Cuba En: http://redalyc.uaemex.mx/src/inicio/Art PdfRed.jsp?iCve=223217613008 (1 de septiembre de 2018) 
Salas, L. (2002). La gerencia social desde la óptica de las organizaciones no gubernamentales. Documento en línea. Disponible en: htpp://org.colombia.blogspot.com/2002 /11/la-gerencia-social-desde-laoptica.html (1 de septiembre de 2018)

Santana, J. y Araujo, Y. (2007). Clima y cultura institucional: $i$ dos constructos para explicar un mismo fenómeno?" Decisiones basadas en el conocimiento $\mathrm{y}$ en el papel social de la empresa: XX Congreso anual de AEDEM, Vol. 1, 2007. pág. 19. En: http://dialnet.unirioja.es /servlet /articulo?codigo=2486886 (18 de septiembre de 2018)

Schneider, B. y Reichers, E. (1983). On the etiology of climates. Personnel Psychology, 36, p. 19-39

Soto, C, (2008). Organizaciones Educativas. Programa de doctorado en ciencias de la educación. Chile: Universidad de La Serena

Taboada, M. (2006). Cultura organizacional y el desempeño docente de instituciones educativas pertenecientes a Fe y Alegría. Lima: Universidad Nacional Federico Villarreal

Taguiri, R. (1969). The concept of Organizational climate. En Taguiri, R. y Litwin, G. (1969). Organizational climate: Explorations of a concept. Boston: Harvard Uni. p. 11-34

Unesco (1995) Innovaciones en la gestión educativa. Experiencias en Brasil, Chile y Venezuela. Santiago, Chile: UNESCO (OREALC)

Xiaofu, P. y Qiwen, Q. (2007). An analysis of the relation between secondary school organizational climate and teacher job satisfaction. Chinese Education and Society, 40(5), 65-77 